\title{
Reliability Analysis of Multi-State Systems based on EUGF Method using Common Cause Failure Components
}

\author{
Jinzhang Jia ${ }^{\mathrm{a}, \mathrm{b}}$, Zhuang Li ${ }^{\mathrm{a}, \mathrm{b}, *}$, Peng Jia ${ }^{\mathrm{a}, \mathrm{b}}$, and Zhiguo Yang ${ }^{\mathrm{c}}$ \\ ${ }^{a}$ School of Safety Science and Engineering, Liaoning University of Engineering and Technology, Fuxin, 123000, China \\ ${ }^{b}$ Institute of Safety Science, Liaoning University of Engineering and Technology, Fuxin, 123000, China \\ ${ }^{c}$ Liaohe Oilfield Construction Co., Ltd., Panjin, 124000, China
}

\begin{abstract}
For the multi-state system, the reliability problem of the common component failure group overlaps under the uncertainty of the component state probability information. Combined with evidence theory, evidence generalized generation function (EUGF) method, and common cause failure theory, the EUGF analysis method for multi-state systems when the common cause failure component groups overlap each other is proposed. The reliability problem of the multi-state system when the common cause failure component group overlaps and the component state probability information has uncertainty is solved. Integrate uncertainty information and common cause failure information in a multi-state system. The reliability of the system and the multi-state system in the case of independent failure of the component is compared. The reliability of the multi-state system in the failure of the common component failure component is lower than the reliability of the component independent failure. The reliability analysis of the multi-state system in the case of the uncertainty of the component state probability information is more in line with the actual engineering situation due to the overlapping of the failed component groups.
\end{abstract}

Keywords: multi-state system (MSS); uncertainty; common cause failure (CCF); reliability; evidence generation function (EUGF)

(Submitted on August 30, 2019; Revised on November 10, 2019; Accepted on December 15, 2019)

(C) 2019 Totem Publisher, Inc. All rights reserved.

\section{Introduction}

Traditional system reliability analysis assumes that the system state is normal and completely ineffective, called a two-state system. However, in practical engineering, due to the complexity of the system structure, it changes in the working environment, efficiency and state. The system has a transitional state process between normal and complete failure. Each state has a state probability corresponding to it. At this time, the relevant theory of the reliability analysis of the traditional two-state hypothesis system will no longer be applicable; therefore, domestic and foreign scholars have proposed the concept of multi-state system (MSS) [1-3]. Since the MSS reliability theory has been proposed, many domestic and foreign scholars have reached many achievements, and the theory is widely used in various fields such as machinery, electric power, aerospace and so on [4-9]. The reliability analysis method of MSS is more complicated than the two-state system. The analysis methods mainly include structural function method, stochastic process method, Monte Carlo, and general generating function (UGF) [10]. Because UGF can overcome the "state dimension explosion" problem that occurs in the calculation process of stochastic process method, it greatly reduces the computational complexity and has been widely used in MSS reliability analysis [11]. The UGF in the traditional MSS theory is based on two basic assumptions [5]: (1) each state probability of each component of the MSS can be accurately measured by probability; (2) the performance state of each component in the MSS is accurately determined.

In the engineering practice, due to the complexity of the system structure and the insufficient number of test samples, accurate values of the state performance level and state probability of each component are difficult to obtain; however, the upper and lower limits of the component state probability are easier to obtain. Many scholars have improved and extended

\footnotetext{
* Corresponding author.

E-mail address: leezhg@126.com
} 
traditional UGF based on some mathematical methods such as fuzzy mathematics, interval mathematics theory, evidence theory, etc., and proposed the fuzzy general generation function [12-14]. The reliability analysis methods such as the interval general generation function [15] and evidence general generation function [16] are used to solve the reliability analysis of complex MSS in the absence of information, lack of data, and incomplete precision. Due to the interval expansion and over-evaluation of the interval UGF method, reliability has certain limitations in accuracy. Wensheng Peng [17] discusses the complexity and data incompleteness of MSS, in which the evidence theory is combined with UGF to apply the EUGF to the reliability analysis of MSS with mixed uncertain information.

When analyzing the reliability of MSS system, it is usually assumed that the failure of each component in the system is independent [18]. However, this assumption often can't reflect the real situation in the actual project, and failure correlation is common. Therefore, in the reliability analysis of complex MSS, the failure correlation is ignored for reliability analysis, which will produce certain error [19]. Common cause failure (CCF) is a common failure related form, which causes the failure of multiple components in the system [18]. Scholars have also carried out a series of research on the problem of common cause failure of MSS. Levitin [20], Liyang Xie, et al. [21] established the reliability analysis model of the nonrepairable MSS with CCF by the improved UGF method. Levitin [22] analyzed the common cause failure in common source failure. Jinyu Zhou and Liyang Xie [23] discussed the occurrence and action mechanism of CCF. Based on the load discretization method and UGF method, a quantitative evaluation model of MSS reliability was established. Jinlei Qin [24] proposed a reliability evaluation method of MSS including CCF in view of mutual exclusion and overlap of CCF component groups. However, none of the above studies have carried out reliability analysis on the case where the common cause failure component groups are superimposed on each other in the case of MSS state probability information uncertainty. At present, how to integrate the uncertainty information and CCF information in MSS still need to be further studied [25]. In this paper, the reliability of MSS components is analyzed by combining the common cause failure information and the uncertainty of component state probability information. It is verified by an example.

The rest of the paper is organized as follows: Section 2 introduces evidence theory to represent theoretical knowledge related to information uncertainty; Section 3 reviews traditional UGF modeling methods and EUGF modeling methods; Section 4 introduces the main form of failure and the modeling method of EUGF when the component groups overlap each other; Section 5 takes the MSS system of fluid-transmitted series-parallel structure as an example to compare the reliability of MSS in the case of independent failure and common cause failure; Section 6 the conclusion and prospect.

\section{Evidence Theory}

The evidence theory was first proposed by Dempster [26-27] and later promoted by Shafer [28], called D-S Evidence Theory (DSET). Evidence theory is defined on a discrete set recognition framework $\Theta$ which is composed of a series of statistically independent and mutually exclusive finite discriminant hypotheses. Recognition framework $\Theta$ contains all possible values of variable $X$, similar to a sample space of variable $X$. A power set $2^{\Theta}$ based on recognition framework $\Theta$ is called trust function. If the recognition frame $\Theta$ contains $n$ elements, then $2^{n}$ is the number of power set elements. The element $X$ in power set $2^{\Theta}$ is called the focal element of recognition frame $\Theta$. The function of focal element difference is represented by the basic probability distribution function $m(X)$ defined on the recognition framework $\Theta$, that is, $m(X)$ : $2^{\Theta} \rightarrow[0,1]$, which is a mapping function of the fixed number $m(X)$ within the focal element $X$ mapped to [0,1], and the value is expressed as the accurate trust degree of the focal element $X$. It meets the following two conditions: (1) when $X=\varphi$, the probability distribution function is 0 ; that is, $m(\varphi)=0 ;(2)$ the probability distribution function the number $m(X)$ is uniform; i.e. $\sum_{X \in 2^{\Theta}} m(X)=1$. For $\forall X \subseteq \Theta$, the exact trust degree of focal element $X$ is $m(X)$; the degree of uncertainty and the agnostic degree of proposition are $m(\Theta)$ [25]. The trust function and likelihood function of event $X$ on recognition framework $\Theta$ are defined as follows:

$$
\begin{aligned}
& \operatorname{Bel}(X)=\sum_{\substack{Y \subseteq X \\
X \subseteq \Theta}} m(Y) \\
& \operatorname{Pl}(X)=\sum_{Y \cap X \neq \Theta} m(Y)
\end{aligned}
$$

In Formula (1), $\operatorname{Bel}(X)$ represents the trust degree of "event $X$ is true", which is regarded as the lower limit of probability measure. $P l(X)$ represents the trust degree of "event $X$ is not false", which is regarded as the upper limit of probability measure [25]. Therefore, the cognitive uncertainty of event $X$ is the value of $P l(X)-\operatorname{Bel}(X)$, which represents a measure of the uncertainty degree of event $X$ caused by insufficient information [29]. Thus, the confidence interval of event 
$X$ is $[\operatorname{Bel}(X), P l(X)]$, which is used to describe the range of possible occurrence of focal element $X$. Figure 1 depicts the relationship between trust and likelihood.

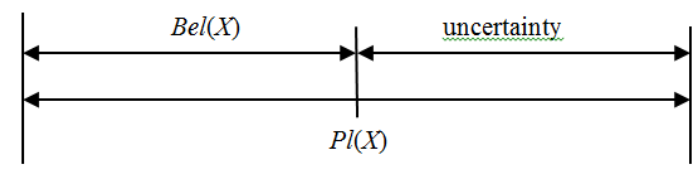

Figure 1. $\operatorname{Bel}(X)$ and $P l(X)$

\section{Evidence General Generation Function (EUGF)}

\subsection{Traditional Universal Generating Function (UGF)}

The classical UGF theory is aimed at discrete random variables and is embodied as a single variable in UGF. The distribution law of discrete random variables consists of two sets: a set $G$ representing the state performance of the system and a set $P$ of probability corresponding to the state. For the component $G^{j}$ of the MSS, if the state $g^{j}$ of each component and the probability $p^{j}$ corresponding to the state are given, the UGF form of the component can be obtained as

$$
U_{j}(Z)=\sum_{i=1}^{k_{j}} p_{i}^{j} z^{g_{i}^{j}}
$$

In Formula (3), $j$ denotes part $j$ and $k$ states of part $j$. The form of UGF of the whole system can be obtained by MSS composed of these components.

$$
U(Z)=\Omega_{\phi}\left(U_{1}(Z), \cdots, U_{n}(Z)\right)=\sum_{i_{1}=1}^{k_{1}} \sum_{i_{2}=1}^{k_{2}} \cdots \sum_{i_{n}=1}^{k_{n}} \prod_{j=1}^{n} p_{i_{j}}^{j} Z^{\phi\left(g_{i}^{1}, \cdots, g_{i_{n}}^{n}\right)}
$$

In Formula (4), $\Omega^{\varphi}$ is a merged operator. It should be pointed out that the global performance of the system determined by the system structure function depends on $Z$. For the cascade structure, the operator is $\Phi_{P}$, and $\Phi_{P}$ is defined as the minimum function.

$$
\Phi_{P}\left(g_{i_{1}}^{1}, g_{i_{2}}^{2}, \cdots, g_{i_{n}}^{n}\right)=\min \left\{g_{i_{1}}^{1}, g_{i_{2}}^{2}, \cdots, g_{i_{n}}^{n}\right\}
$$

For parallel structures, the operator is $\Phi_{S}$, and $\Phi_{S}$ is defined as sum function.

$$
\Phi_{P}\left(g_{i_{1}}^{1}, g_{i_{2}}^{2}, \cdots, g_{i_{n}}^{n}\right)=\sum_{j=1}^{n} g_{i_{n}}^{n}
$$

The case where the relationship between the system performance level and the demand $w$ is usually determined by using the MSS state to satisfy the index $r_{i}$, that is, $r_{i}=g_{i}-w$. State $i$ is an acceptable state if and only if $r_{i} \geq 0$. Generally, the reliability of the MSS can be defined as the probability that the performance level of the MSS is greater than the demand, ie.

$$
R(w)=\sum_{r_{i} \geq 0} p_{i}
$$

For a given $U(Z)$ and $w$, the steady state reliability of the MSS that can be obtained by applying the operator $\delta_{R}$, ie.

$$
R(w)=\delta_{R}(U(Z), w)=\delta_{R}\left(\sum_{i=1}^{K} p_{i} g Z^{g_{i}}, w\right)=\sum_{i=1}^{K} p_{i} g \alpha_{i}
$$




$$
\alpha_{i}=\left\{\begin{array}{l}
1, r_{i} \geq 0 \\
0, r_{i}<0
\end{array}\right.
$$

\subsection{Evidence General Generation Function}

In the EUGF model, the individual performance state information of system or component is given in interval form, and the uncertainty quantization of each performance state is the basic trust distribution function in the evidence theory. Similarly, for the component $G^{j}$ of the MSS, there are a total of $n$ components, and the performance state space can be $g_{j}=\left\{g_{1}, g_{2}, \cdots, g_{n}\right\}$, and the corresponding state probability interval is, $\left[P_{j, 1}, P_{j, 1}\right], \cdots,\left[P_{j, m}, P_{j, m}\right]$. The interval $\left[P_{j, 1}, P_{j, 1}\right]$ is approximated as follows to obtain an accurate mass function.

$$
m(S)=\left\{\begin{array}{lc}
\underline{P}_{j, m}, & S=g_{i, m} \\
1-\sum_{j=1}^{m_{j}} \underline{P}_{j, m}, & S=g_{i, 1}, g_{i, 2}, \cdots, g_{i, m} \\
0, & \text { else }
\end{array}\right.
$$

In Formula (10), $S$ is a set containing all states of component $j$, and $m(S)$ is a mass function of state space $g_{j}$, that is, a mapping of each element state probability interval to $[0,1]$. According to the mass function $m(S)$ mapping method of Formula (10), the focal element of component $j$ can be expressed as $[g]_{j, 1},[g]_{j, 2}, \cdots,[g]_{j, k_{j}}$. Suppose the MSS consists of $n$ components. When the component performance level $g_{j}$ can be expressed as $[g]_{j}\left(1 \leq i \leq n,[g]_{j} \subset g_{j}\right)$, the relationship between the MSS structure before and after mapping is:

$$
\phi\left([g]_{1}, \cdots,[g]_{n}\right)=\left\{\phi\left(g_{1}, \cdots, g_{n}\right) \mid g_{j} \in[g]_{j}\right\}
$$

If $m_{i j}$ is the mass of the first state focal element $[g]_{j, i}\left(1 \leq i \leq k_{j}, 1 \leq j \leq n\right)$, then the state information of each component $j$ can be transformed into an UGF, that is, EUGF. The EUGF of component $j$ with $k_{j}$ performance state is defined as:

$$
U_{j}^{b}(Z)=\sum_{i=1}^{k_{j}} p_{j, m} z^{[g]_{j, m}}
$$

The EUGF form of the MSS consisting of $n$ components is:

$$
U^{b}(Z)=\Omega_{\phi}\left(U_{1}^{b}(Z), \cdots, U_{n}^{b}(Z)\right)=\sum_{i_{1}=1}^{k_{1}} \sum_{i_{2}=1}^{k_{2}} \cdots \sum_{i_{n}=1}^{k_{n}} \prod_{j=1}^{n} p_{j, i_{j}} Z^{\left.\phi[g]_{1, j}, \cdots,[g]_{n, j_{n}}\right)}
$$

The composite operator in EUGF has the same meaning as the series-parallel structure of the traditional general generating function.

When the system state performance level requirement is $w,(14)$ and (15) are the likelihood function of event $X$ and the operation operator of the trust function, respectively.

$$
\begin{gathered}
1_{w}^{+}= \begin{cases}1, \Phi\left([g]_{1}, \cdots,[g]_{n}\right) \cap w \neq \phi \\
0, & \text { else }\end{cases} \\
1_{w}^{-}= \begin{cases}1, \Phi\left([g]_{1}, \cdots,[g]_{n}\right) \subseteq w \\
0, & \text { else }\end{cases}
\end{gathered}
$$

In Formula (14), $\Phi\left([g]_{1}, \cdots,[g]_{n}\right) \bigcap w \neq \varphi$ indicates that at least one element in the interval value performance is not 
less than the system performance requirement $w$. In Formula (15), $\Phi\left([g]_{1}, \cdots,[g]_{n}\right) \subseteq w$ indicates that all elements in the interval value performance are not less than the system performance requirement $w$.

When the system state performance level satisfies the system demand $w$, the upper limit $P l(w)$ and the lower limit $\operatorname{Bel}(w)$ of the system reliability can be calculated by (16) and (17), respectively.

$$
\begin{gathered}
P l(w)=1_{w}^{+}(U(Z))=1_{w}^{+}\left(\sum_{i_{1}=1}^{k_{1}} \sum_{i_{2}=1}^{k_{2}} \cdots \sum_{i_{n}=1}^{k_{n}} \prod_{j=1}^{n} p_{j, i_{j}} Z^{\Phi}\right)=\sum_{i_{1}=1}^{k_{1}} \sum_{i_{2}=1}^{k_{2}} \cdots \sum_{i_{n}=1}^{k_{n}} \prod_{j=1}^{n} p_{j, i_{j}} 1_{w}^{+} \\
\operatorname{Bel}(w)=1_{w}^{-}(U(Z))=1_{w}^{-}\left(\sum_{i_{1}=1}^{k_{1}} \sum_{i_{2}=1}^{k_{2}} \cdots \sum_{i_{n}=1}^{k_{n}} \prod_{j=1}^{n} p_{j, i_{j}} Z^{\Phi}\right)=\sum_{i_{1}=1}^{k_{1}} \sum_{i_{2}=1}^{k_{2}} \cdots \sum_{i_{n}=1}^{k_{n}} \prod_{j=1}^{n} p_{j, i_{j}} 1_{w}^{-}
\end{gathered}
$$

\section{EUGF When the Common Component Fails to Overlap Each Other}

In the case of common cause failure and EUGF fusion, based on the following basic assumptions:

(1) It is assumed that the component or system has a finite number of discrete states and the system is an irreparable system.

(2) Assume that the state probability of a component or system is uncertain.

(3) Assuming that the load is greater than the component's endurance or external impact, the load on all components in the common cause group will exceed its strength limit, causing all the same components in the common cause group to fail simultaneously.

According to the source of the common cause failure, it can be divided into external causes and internal causes. The common cause of internal causes fails, usually due to the failure of one component itself, causing other components to fail. Therefore, a common cause failure caused by internal causes is called propagation failure. Some components only have their own failure state, and will not cause failure of other components. In a multi-state component with propagation failure, there may be multiple different components that cause additional components to fail. The common cause failure component caused by each component together forms a plurality of common cause failure component groups. These common cause failure component groups can be independent of each other, and some components can overlap each other. Under the combined effect of component failure and common cause failure, the performance level of the system will gradually decrease. State 0' represents the failure state caused by common cause failure, state 0 represents the complete failure state caused by non-common cause failure, and the performance level of component $j$ in common cause failure state 0 ' can be expressed as $g_{j 0^{\prime}}$. Compared with the propagation failure of components, the failure state 0 of components itself is also called local failure. In order to be consistent with the performance level of failure state 0 , it is usually set to $g_{j 0^{\prime}}=0$. When component $j$ can cause common cause failure of other components with a certain probability $P_{j 0^{\prime}}$, the EUGF of the component can be given as Formula (18).

$$
U_{j}^{c}(Z)=\sum_{j=1}^{k_{j}} p_{j, m} Z^{[g]_{j, m}}+p_{j, 0^{\prime}} Z^{[g]_{j, 0^{\prime}}}
$$

For any component $j$ in the system, the corresponding condition EUGF can be expressed as.

$$
U_{j}^{\prime c}(Z)=\sum_{j=1}^{k_{j}} \frac{p_{j, m}}{1-p_{j, 0^{\prime}}} Z^{[g]_{j, m}}
$$

Assuming that there are $b \leq n$ components in the system that can cause CCF independently of each other, it is recorded as a component vector where $\alpha=\{\alpha(1) \cdots \alpha(r) \alpha(s)\}, r \in[1, t]$, and the probability that each component is in a state of 0 '. This causes CCF, which is denoted as $P_{\alpha(r) 0^{\prime}}$. In the whole system, these components cause multiple groups of CCF in the way of combination, so the space number of the whole combination can reach $2^{b}$. For any combinations of $0 \leq \delta \leq 2^{b}-1$, 
the CCF caused by $\alpha(r)$ the component needs to satisfy the conditions.

$$
\varepsilon(r)=\bmod _{2}\left[\frac{\delta}{2^{r-1}}\right]=1,1 \leq r \leq s
$$

From $\delta=0$ to $\delta=2^{b}-1$, the set of components corresponding to each combination $\delta$ can be obtained. The probability of CCF caused by each combination $\delta$ is determined by the components contained in the combination.

$$
\phi_{\delta}=\prod_{r=1}^{t} p_{\alpha(r) 0^{\prime}}^{\varepsilon(r)}\left(1-p_{\alpha(r) 0^{\prime}}\right)^{1-\varepsilon(r)}, 0 \leq \delta \leq 2^{b}-1
$$

If the set of components $\alpha(r)$ that can cause other components $S_{\alpha(r)}$ to fail, it becomes the set of components that belong to the common cause failure corresponding to any combination of $\delta$. The set of components that belong to the same combination is the set of components that can cause other components to fail.

$$
\mu_{\delta}=\bigcup_{r=1}^{t} S_{\alpha(r)}^{\prime}
$$

Among them:

$$
\begin{cases}S_{\alpha(r)} \cup\{\alpha(r)\}, & \varepsilon(r)=1 \\ S_{\alpha(r)}^{\prime}=\phi, & \varepsilon(r)=0\end{cases}
$$

According to Formula (19), the EUGF of components can be determined and the EUGF expression $\mu_{\delta}(Z)$ of the whole system under specified combination $\delta$ can be established in combination with the physical structure of the system. If $\mu_{\delta} \neq \varphi$, the EUGF expression in which the corresponding component is in CCF state should be replaced $\rho(Z)=Z^{0}$; otherwise, no replacement will be carried out. By using the theorem of total probability formula and combining with (21), the EUGF expression of the whole system under the action of CCF is as follows.

$$
U^{c}(Z)=\sum_{\delta=0}^{2^{b}-1} u_{\delta}(z) \cdot \phi_{\delta}
$$

According to the above formula, combined with (16) and (17), the reliability of the system under CCF conditions can be calculated.

\section{Example Analysis}

Assume an MSS series-parallel structure system consisting of subsystem 1 and subsystem 2, as shown in Figure 2. MSS can be thought of as a fluid transfer system structure, such as a pump set. Subsystem 1 consists of two parts, $A$ and $B$, which have four states, namely, good state, intermediate state, failure state, and propagation failure state. Subsystem 2 consists of three states, components, $C, D$, and $E$, in good condition, intermediate state, and failed state. Component $A$ causes $C C F$ to occur in component $C$, forming a common cause failure group. Component $B$ causes $C C F$ to occur in component $D$ and component $E$ forms another common cause failure group. In this system, it is assumed that the state performance of all components is an exact value, and the physical meaning of the specific performance level of each component is ignored. The probability of each state is shown in Table 1.

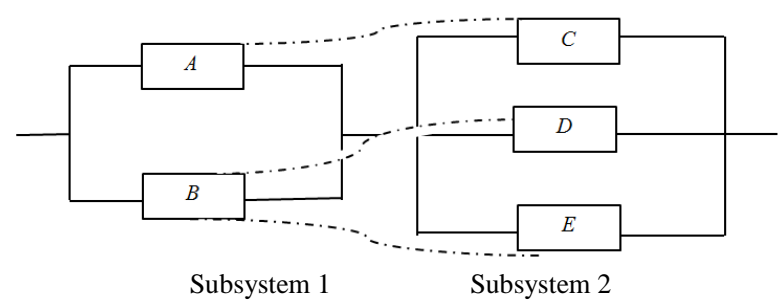

Figure 2. A fluid transmission system 
Table 1. State performance and state probability table of fluid transmission system in component overlapping common cause failure

\begin{tabular}{|c|c|c|c|c|c|c|c|c|}
\hline component $j$ & $X_{j 1}$ & {$\left[p_{j 1}\right]$} & $X_{j 2}$ & {$\left[p_{j 2}\right]$} & $X_{j 3}$ & {$\left[p_{j 3}\right]$} & $X_{j 0}{ }^{\prime}$ & {$\left[p_{j 0^{\prime}}\right]$} \\
\hline$A$ & 0 & {$[0.080,0.090]$} & 1.5 & {$[0.090,0.100]$} & 3 & {$[0.795,0.805]$} & 0 & {$[0.015,0.025]$} \\
\hline$B$ & 0 & {$[0.093,0.103]$} & 1 & {$[0.092,0.102]$} & 1.5 & {$[0.790,0.800]$} & $0^{\prime}$ & {$[0.020,0.030]$} \\
\hline$C$ & 0 & {$[0.096,0.106]$} & 1 & {$[0.095,0.105]$} & 2 & {$[0.794,0.804]$} & - & - \\
\hline$D$ & 0 & {$[0.095,0.105]$} & 1.5 & {$[0.190,0.200]$} & 2 & {$[0.695,0.705]$} & - & - \\
\hline$E$ & 0 & {$[0.090,0.110]$} & 0.5 & {$[0.185,0.195]$} & 1 & {$[0.705,0.715]$} & - & - \\
\hline
\end{tabular}

\subsection{MSS Reliability When Component Common Cause Failure Groups Overlap}

According to Formula (10), Formula (12), Formula (18), the EUGF of each component under CCF condition is obtained.

$$
\begin{aligned}
& U_{A}^{c}(Z)=0.08 Z^{0}+0.09 Z^{1.5}+0.795 Z^{3}+0.015 Z^{0^{\prime}}+0.02 Z^{0,1.5,3} \\
& U_{B}^{c}(Z)=0.093 Z^{0}+0.092 Z^{1}+0.790 Z^{1.5}+0.02 Z^{0^{\prime}}+0.005 Z^{0,1,1.5} \\
& U_{C}^{c}(Z)=0.096 Z^{0}+0.095 Z^{1}+0.794 Z^{2}+0.015 Z^{0,1,2} \\
& U_{D}^{c}(Z)=0.095 Z^{0}+0.190 Z^{1.5}+0.695 Z^{2}+0.015 Z^{0,1.5,2} \\
& U_{E}^{c}(Z)=0.090 Z^{0}+0.185 Z^{0.5}+0.705 Z^{1}+0.015 Z^{0,0.5,1}
\end{aligned}
$$

According to Formula (19), the condition EUGF corresponding to each component is obtained.

$$
\begin{aligned}
& U_{A}^{\prime c}(Z)=0.0812 Z^{0}+0.0913 Z^{1.5}+0.8071 Z^{3}+0.0204 Z^{0,1.5,3} \\
& U_{B}^{\prime c}(Z)=0.0949 Z^{0}+0.0938 Z^{1}+0.8061 Z^{1.5}+0.0052 Z^{0,1,1.5} \\
& U_{C}^{\prime c}(Z)=0.096 Z^{0}+0.095 Z^{1}+0.794 Z^{2}+0.015 Z^{0,1,2} \\
& U_{D}^{\prime c}(Z)=0.095 Z^{0}+0.190 Z^{1.5}+0.695 Z^{2}+0.015 Z^{0,1.5,2} \\
& U_{E}^{\prime c}(Z)=0.090 Z^{0}+0.185 Z^{0.5}+0.705 Z^{1}+0.015 Z^{0,0.5,1}
\end{aligned}
$$

In this case, there are two components that cause $\mathrm{CCF}$, i.e. $\alpha(1)=A, \alpha(2)=B$, according to the components that cause CCF, they are respectively, $S_{\alpha(1)}=\{C\}, S_{\alpha(2)}=\{D\}$. The values of the four possible combinations of variables that trigger CCF corresponding to the two components are listed in Table 2.

Table 2. Values of variables in common cause failure combination

\begin{tabular}{|c|c|c|c|c|}
\hline$\delta$ & $\varepsilon(1)$ & $\varepsilon(2)$ & $\varphi_{\delta}$ & $\mu_{\delta}$ \\
\hline 0 & 0 & 0 & 0.905 & $\phi$ \\
\hline 1 & 1 & 0 & 0.052 & $\{A, C\}$ \\
\hline 2 & 0 & 1 & 0.041 & $\{B, D, E\}$ \\
\hline 3 & 1 & 1 & 0.002 & $\{A, C\} \cup\{B, D, E\}$ \\
\hline
\end{tabular}

MSS belongs to series-parallel structure, that is, subsystem 1 is composed of $A$ and $B$ in parallel, subsystem 2 is composed of $C, D$ and $E$ in parallel, and the total system is composed of subsystem 1 and subsystem 2 in series. According to Formula (5), (6) EUGF under $\delta$ different combinations, namely.

$$
\mu_{\delta}(Z)=\left(U_{A}^{\prime c}(Z) \underset{\text { sum }}{\otimes} U_{B}^{\prime c}(Z)\right) \underset{\text { min }}{\otimes}\left(U_{C}^{\prime c}(Z) \underset{\text { sum }}{\otimes} U_{D}^{\prime c}(Z) \underset{\text { sum }}{\otimes} U_{E}^{\prime c}(Z)\right)
$$

When $\delta=0, \mu_{\delta}=\phi$, none of the components in Formula (25) is replaced by $\rho(Z)$, which is calculated.

$$
\begin{aligned}
& \mu_{0}(Z)=\left(0.0812 Z^{0}+0.0913 Z^{1.5}+0.8071 Z^{3}+0.0204 Z^{0,1.5,3}\right. \\
& \left.\underset{\text { sum }}{\otimes} 0.0949 Z^{0}+0.0938 Z^{1}+0.8061 Z^{1.5}+0.0052 Z^{0,1,1.5}\right) \underset{\min }{\otimes}\left(0.096 Z^{0}+0.095 Z^{1}\right. \\
& +0.794 Z^{2}+0.015 Z^{0,1,2} \underset{\text { sum }}{\otimes} 0.095 Z^{0}+0.190 Z^{1.5}+0.695 Z^{2}+0.015 Z^{0,1.5,2} \underset{\text { sum }}{\otimes} \\
& \left.0.090 Z^{0}+0.185 Z^{0.5}+0.705 Z^{1}+0.015 Z^{0,0.5,1}\right)=0.0085 Z^{0}+0.0167 Z^{0.5}+0.0732 Z \\
& +0.0804 Z^{1.5}+0.0703 Z^{2}+0.0433 Z^{2.5}+0.1834 Z^{3}+0.0265 Z^{3.5} \\
& +0.1124 Z^{4}+0.3745 Z^{4.5}+0.0073 Z^{1,1.5,2}+0.0035 Z^{1.5,3,4.5}
\end{aligned}
$$


When $\delta=1, \mu_{\delta}=\{A, C\}$, the EUGF corresponding to the components $A$ and $C$ in Equation (25) is replaced by $\rho(Z)$.

$$
\begin{aligned}
& \mu_{1}(Z)=\left(\left(Z^{0}\right) \underset{\text { sum }}{\otimes} 0.0949 Z^{0}+0.0938 Z^{1}+0.8061 Z^{1.5}+0.0052 Z^{0,1,1.5}\right) \\
& \underset{\min }{\otimes}\left(\left(Z^{0}\right) \underset{\text { sum }}{\otimes} 0.095 Z^{0}+0.190 Z^{1.5}+0.695 Z^{2}+0.015 Z^{0,1.5,2} \underset{\text { sum }}{\otimes} 0.090 Z^{0}+0.185 Z^{0.5}\right. \\
& \left.+0.705 Z^{1}+0.015 Z^{0,0.5,1}\right)=0.1036 Z^{0}+0.0163 Z^{0.5}+0.1497 Z^{1}+0.7157 Z^{1.5} \\
& +0.0015 Z^{0,0.5,1}+0.0005 Z^{0,1,1.5}+0.0011 Z^{0,1.5,1.5}+0.0005 Z^{0,1,1} \\
& +0.0003 Z^{0.5,1,1}+0.0023 Z^{0.5,1.5,1.5}+0.0085 Z^{1,1.5,1.5}
\end{aligned}
$$

When $\delta=2, \mu_{\delta}=\{B, D, E\}$, The EUGF corresponding to the components $B, D$ and $E$ in Formula (25) is replaced by $\rho(Z)$.

$$
\begin{aligned}
& \mu_{2}(Z)=\left(0.0812 Z^{0}+0.0913 Z^{1.5}+0.8071 Z^{3}+0.0204 Z^{0,1.5,3} \underset{\text { sum }}{\otimes}\left(Z^{0}\right)\right) \underset{\min }{\otimes}\left(0.096 Z^{0}\right. \\
& \left.+0.095 Z^{1}+0.794 Z^{2}+0.015 Z^{0,1,2} \underset{\text { sum }}{\otimes}\left(Z^{0}\right) \underset{\text { sum }}{\otimes}\left(Z^{0}\right)\right)=0.1694 Z^{0}+0.0854 Z^{1}+ \\
& 0.0725 Z^{1.5}+0.6408 Z^{2}+0.0014 Z^{0,1,1.5}+0.0019 Z^{0,1,1}+0.0162 Z^{0,1.5,2}+0.0124 Z^{0,1,2}
\end{aligned}
$$

When $\delta=3, \mu_{\delta}=\{A, C\} \bigcup\{B, D, E\}$, The EUGF of all components in Formula (25) is replaced by $\rho(Z)$.

$$
\mu_{3}(Z)=\left(\left(Z^{0}\right) \underset{\text { sum }}{\otimes}\left(Z^{0}\right)\right) \underset{\min }{\otimes}\left(\left(Z^{0}\right) \underset{\text { sum }}{\otimes}\left(Z^{0}\right) \underset{\text { sum }}{\otimes}\left(Z^{0}\right)\right)=Z^{0}
$$

According to Formula (21), the EUGF expression of the whole MSS can be obtained as follows.

$$
\begin{aligned}
& U^{c}(Z)=\sum_{\delta=0}^{3} u_{\delta}(z) \cdot \phi_{\delta}=0.202 Z^{0}+0.0159 Z^{0.5}+0.0775 Z^{1}+0.1130 Z^{1.5} \\
& +0.0899 Z^{2}+0.0392 Z^{2.5}+0.1669 Z^{3}+0.0242 Z^{3.5}+0.1024 Z^{4}+0.3389 Z^{4.5} \\
& +0.0001 Z^{0,0.5,1}+0.0001 Z^{0,1,1.5}+0.0001 Z^{0,1.5,1.5}+0.0001 Z^{0,1,1}+0.0007 Z^{0,1.5,2} \\
& +0.0005 Z^{0,1,2}+0.0001 Z^{0.5,1.5,1.5}+0.0004 Z^{1,1.5,1.5}+0.0066 Z^{1.1 .5 .2}+0.0032 Z^{1.5,3,4.5}
\end{aligned}
$$

When the demand performance level of the system is $w=2$, the reliability interval of the system is (14)-(17).

$$
R=[\operatorname{Bel}(w), P l(w)]=[0.7615,0.7725]
$$

\subsection{MSS Reliability When Components Fail Independently}

When the components of MSS fail independently, the probability of each component's state is shown in Table 3 , and the structure of the MSS system is shown in Figure 3.

Table 3. State performance and state probability table of fluid transmission system in component independent failure

\begin{tabular}{|c|c|c|c|c|c|c|}
\hline Tamponent $j$ & $X_{j 1}$ & {$\left[p_{j 1}\right]$} & $X_{j 2}$ & {$\left[p_{j 2}\right]$} & $X_{j 3}$ & {$\left[p_{j 3}\right]$} \\
\hline$A$ & 0 & {$[0.095,0.105]$} & 1.5 & {$[0.090,0.100]$} & 3 & {$[0.795,0.805]$} \\
\hline$B$ & 0 & {$[0.113,0.123]$} & 1 & {$[0.092,0.102]$} & 1.5 & {$[0.790,0.800]$} \\
\hline$C$ & 0 & {$[0.096,0.106]$} & 1 & {$[0.095,0.105]$} & 2 & {$[0.794,0.804]$} \\
\hline$D$ & 0 & {$[0.095,0.105]$} & 1.5 & {$[0.190,0.200]$} & 2 & {$[0.695,0.705]$} \\
\hline$E$ & 0 & {$[0.090,0.110]$} & 0.5 & {$[0.185,0.195]$} & 1 & {$[0.705,0.715]$} \\
\hline
\end{tabular}

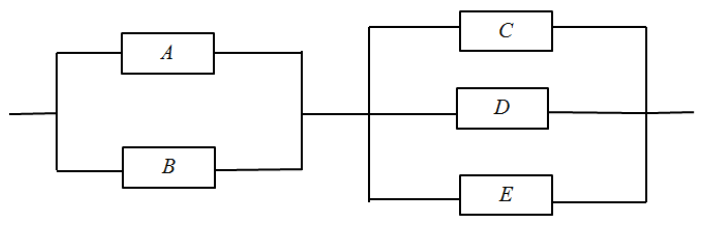

Subsystem 1

Subsystem 2

Figure 3. A fluid transmission system fails independently 
According to Formula (10) and Formula (12), the EUGF of each component under the condition of independent failure is obtained.

$$
\begin{aligned}
& U_{A}^{b}(Z)=0.095 Z^{0}+0.09 Z^{1.5}+0.795 Z^{3}+0.015 Z^{0^{\prime}}+0.02 Z^{0,1.5,3} \\
& U_{B}^{b}(Z)=0.113 Z^{0}+0.092 Z^{1}+0.790 Z^{1.5}+0.005 Z^{0,1,1.5} \\
& U_{C}^{b}(Z)=0.096 Z^{0}+0.095 Z^{1}+0.794 Z^{2}+0.015 Z^{0,1,2} \\
& U_{D}^{b}(Z)=0.095 Z^{0}+0.190 Z^{1.5}+0.695 Z^{2}+0.015 Z^{0,1.5,2} \\
& U_{E}^{b}(Z)=0.090 Z^{0}+0.185 Z^{0.5}+0.705 Z^{1}+0.015 Z^{0,0.5,1}
\end{aligned}
$$

According to the Formulas (5) and (6), the EUGF is obtained when the components of the system fail independently.

$$
\begin{aligned}
& U(Z)=\left(U_{A}^{b}(Z) \underset{\text { sum }}{\otimes} U_{B}^{b}(Z)\right) \underset{\min }{\otimes}\left(U_{C}^{b}(Z) \underset{\text { sum }}{\otimes} U_{D}^{b}(Z) \underset{\text { sum }}{\otimes} U_{E}^{b}(Z)\right)= \\
& \left(0.095 Z^{0}+0.09 Z^{1.5}+0.795 Z^{3}+0.02 Z^{0,1.5,3} \underset{\text { sum }}{\otimes} 0.113 Z^{0}+0.092 Z^{1}+\right. \\
& \left.0.790 Z^{1.5}+0.005 Z^{0,1,1.5}\right) \underset{\min }{\otimes}\left(0.096 Z^{0}+0.095 Z^{1}+0.794 Z^{2}+0.015 Z^{0,1,2}\right. \\
& \underset{\text { sum }}{\otimes} 0.095 Z^{0}+0.190 Z^{1.5}+0.695 Z^{2}+0.015 Z^{0,1.5,2} \underset{\text { sum }}{\otimes} 0.090 Z^{0}+0.185 Z^{0.5} \\
& \left.+0.705 Z^{1}+0.015 Z^{0,0.5,1}\right)=0.0113 Z^{0}+0.0195 Z^{0.5}+0.0714 Z^{1}+0.0805 Z^{1.5} \\
& +0.0771 Z^{2}+0.0493 Z^{2.5}+0.1808 Z^{3}+0.0293 Z^{3.5}+0.1064 Z^{4}+0.3639 Z^{4.5} \\
& +0.0072 Z^{1,1.5,2}+0.0033 Z^{1.53,4.5}
\end{aligned}
$$

When the demand performance level of the system is $w=2$, the reliability interval of the system is (14)-(17).

$$
R^{\prime}=[0.8068,0.8173]
$$

It can be seen from the calculation results that the reliability of the MSS under the CCF due to the superposition of the failed component groups is lower than the reliability of the independent failure of the MSS components, indicating the reliability of the MSS in the overlap of the components in the common cause failure component group. Neglecting the MSS common cause failure conditions for reliability analysis and actual conditions will produce certain errors. Considering the common cause of failure, the reliability analysis of the failure component groups superimposed on each other is more in line with the actual situation of the project. Common cause failure is one of the important reasons for reducing the reliability of MSS. Therefore, corresponding protection measures are taken for components that are prone to cause common cause failure, which reduces the occurrence of common cause failure and the superposition of common cause failure component groups, thereby improving MSS. Reliability.

\section{Conclusions}

In this paper, the reliability problem of MSS due to the overlapping of component parts under the uncertainty of component state probability information is analyzed. The analysis method of MSS reliability based on EUGF is proposed. The uncertainty information and common cause failure information are presented. MSS is fused. Compared with the reliability of the MSS components, the reliability of the MSS common failure component groups is lower than the reliability of the MSS when they overlap each other, which has a certain impact on the reliability of the MSS. The MSS reliability is the error generated in the calculation of the component's independent failure. The common cause failure is one of the reasons for the reliability of the MSS. In actual problems, the component state probability information often has uncertainty. The common component failure component groups can be mutually exclusive or overlapped. Therefore, it is more realistic to use the EUGF for MSS reliability analysis when the common component failure component groups are superimposed on each other. It has certain guiding significance in engineering practice. The next step is to consider the MSS unit and subsystem in a continuous repairable state, combining the stochastic process with the EUGF for common cause failure reliability analysis.

\section{References}

1. Y. K. Gu and J. Li, "Multi-State System Reliability: A New and Systematic Review," Procedia Engineering, Vol. 29, pp. 531536,2012

2. A. Lisnianski, D. Elmakias, D. Laredo, and H. B. Haim, "A Multi-State Markov Model for a Short-Term Reliability Analysis of 
a Power Generating Unit,” Reliability Engineering and System Safety, Vol. 98, No. 1, pp. 1-6, 2012

3. Y. Massim, A. Zeblah, M. Benguediab, A. Ghouraf, and R. Meziane, "Reliability Evaluation of Electrical Power Systems Including Multi-State Considerations," Electrical Engineering, Vol. 88, No. 2, pp. 109-116, 2006

4. J. F. Caete and J. H. Saleh, "Beyond Reliability Multi-State Failure Analysis of Satellite Subsystems: A Statistical Approach," Reliability Engineering and System Safety, Vol. 95, pp. 311-322, 2010

5. J. K. Wayer, J. F. Castet, and J. H. Saleh, "Spacecraft Attitude Control Subsystem: Reliability, Multi-State Analyses, and Comparative Failure Behavior in LEO and GEO," Acta Astronautica, Vol. 85, pp. 83-92, 2013

6. S. Y. Kim, J. F. Castet, and J. H. Saleh, "Spacecraf Telectrical Power Subsystem: Failure Behavior, Reliability, and Multi-State Failure Analyses," Reliability Engineering and System Safety, Vol. 98, pp. 55-65, 2012

7. D. N. Chen and C. Y. Yao, "Reliability Analysis of Polymorphic Systems based on Fuzzy Bayesian Network and its Application in Hydraulic Systems," Journal of Mechanical Engineering, Vol. 48, No. 16, pp. 175-183, 2012

8. Y. Liu, X. Y. Liu, and X. H. Zhang, "Reliability Modeling and Optimization of Multi-State System Considering Dynamic Load Distribution Mechanism,” Journal of Mechanical Engineering, Vol. 52, No. 6, pp. 197-205, 2016

9. Y. F. Li and E. Zio, "A Multi-State Model for the Reliability Assessment of a Distributed Generation System via Universal Generating Function," Reliability Engineering and System Safety, Vol. 106, pp. 28-36, 2012

10. W. C. A. Yeh, "Simple Universal Generating Function Method for Estimating the Reliability of General Multi-State Node Networks," IIE Transactions, Vol. 41, No. 1, pp. 3-11, 2008

11. Y. Liu, "Reliability Modeling and Maintenance Decision for Multi-State Complex Systems," Ph.D thesis, University of Electronic Science and Technology, Chengdu, China, 2010

12. Y. Ding and A. Lisnianski, "Fuzzy Universal Generating Functions for Multi-State System Reliability Assessment," Fuzzy Sets and Systems, Vol. 159, No. 3, pp. 307-324, 2008

13. Y. Ding, M. J. Zuo, A. Lisnianski, and Z. G. Tian, "Fuzzy Multi-State Systems: General Definitions, and Performance Assessment," IEEE Transactions on Reliability, Vol. 57, No. 4, pp. 589-594, 2008

14. Y. Liu and H. Z. Huang, "Reliability Assessment for Fuzzy Multi-State Systems," International Journal of Systems Science, Vol. 41, No. 4, pp. 365-379, 2010

15. S. Destercke and M. Sallak, "An Extension of Universal Generating Function in Multi-State Systems Considering Epistemic Uncertainties," IEEE Transactions on Reliability, Vol. 62, No. 2, pp. 504-514, 2013

16. C. Li, X. Chen, X. Yi, and J. Y. Tao, "Interval-Valued Reliability Analysis of Multi-State Systems," IEEE Transactions on Reliability, Vol. 60, No. 1, pp. 323-330, 2011

17. W. S. Peng, J. G. Zhang, J. Y. Zhang, et al., "EUGF Analysis Method for Reliability of Hybrid Uncertain Polymorphic Systems," Systems Engineering and Electronic Technology, Vol. 39, No. 12, pp. 2863-2868, 2017

18. C. Y. Li, "Research on Reliability Analysis and Optimization Design Method based on Multi State System Theory," Ph.D thesis, National University of Defense Science and Technology, Changsha, China, 2010

19. J. H. Mi, Y. F. Li, W. W. Peng, and H. Z. Huang, "Reliability Analysis of Multi-State System with Common Cause Failure based on Bayesian Networks," Maintenance and Reliability, Vol. 15, No. 2, pp. 169-175, 2013

20. G. Levitin, "Incorporating Common-Cause Failures into Nonrepairable Multistate Series-Parallel System Analysis," IEEE Transactions on Reliability, Vol. 50, No. 4, pp. 380-388, 2001

21. J. Y. Zhou, L. Y. Xie, and X. M. Wang, "Common Cause Failure Analysis and Reliability Model for Multi-State Systems," Journal of Mechanical Engineering, Vol. 41, No. 6, pp. 66-70, 2005

22. G. Levitin, "Common Supply Failures in Linear Multi-State Sliding Window Systems," Reliability Engineering and System Safety, Vol. 82, pp. 55-62, 2003

23. J. Y. Zhou and L. Y. Xie, "Common Cause Failure Mechanism and Quantitative Analysis of Multi-State Systems," Journal of Mechanical Engineering, Vol. 44, No. 10, pp. 77-82, 2008

24. J. L. Qin, "Research on Reliability Evaluation Method of Complex Multi-State System," Ph.D thesis, North China Electric Power University, Beijing, China, 2016

25. J. H. Mi, "Reliability Analysis and Evaluation of Complex Systems under Cognitive Uncertainty," Ph.D thesis, University of Electronic Science and Technology, Chengdu, China, 2017

26. A. P. Dempster, "Upper and Lower Probabilities Induced by a Multivalued Mapping," The Annals of Mathematical Statistics, Vol. 38, No. 2, pp. 325-339, 1967

27. A. P. Dempster, "A Generalization of Bayesian Inference," Journal of the Royal Statistical Society, Series B (Methodological), Vol. 30, No. 2, pp. 205-247, 1968

28. G. Shafer, “A Mathematical Theory of Evidence, Princeton,” Princeton University Press, USA, 1976

29. F. Aguirre, M. Sallak, and W. Schön, "Construction of Belief Function from Statistical Data about Reliability under Epistemic Uncertainty," IEEE Transactions on Reliability, Vol. 62, No. 3, pp. 555-568, 2013

Jinzhang Jia is a professor of the Liaoning University of Engineering and Technology. His research interests include safety science, safety technology, mine ventilation and disaster prevention, system reliability theory.

Zhuang $\mathbf{L i}$ is an Ph.D candidate \& engineer of the Liaoning University of Engineering and Technology. His research interests include System reliability theory, safety science. 